\title{
Effects of epinephrine on hemodynamic changes during cardiopulmonary resuscitation in a neonatal piglet model
}

\author{
Michael Wagner ${ }^{1,2}$, Po-Yin Cheung ${ }^{2,3}$, Elliott S. Li ${ }^{2,3}$, Tze-Fun Lee ${ }^{2,3}$, Min Lü ${ }^{2,3}$, Megan O'Reilly ${ }^{2,3}$, Monika Olischar ${ }^{1}$ and \\ Georg M. Schmölzer ${ }^{2,3}$
}

BACKGROUND: Asphyxia is the most common reason for newborns to fail to make a successful fetal-to-neonatal transition. There is currently a lack of data evaluating hemodynamic effects of epinephrine during neonatal cardiopulmonary resuscitation.

METHODS: Twenty-four newborn piglets were exposed to asphyxia. Thereafter, positive pressure ventilation was commenced for $30 \mathrm{~s}$, followed by chest compressions (CC). Piglets were randomized into three experimental groups: 3:1 compression:ventilation ratio; CC during sustained inflation (SI) at a rate of 90 CC per minute, or CC during SI at a rate of 120 CC per minute. Epinephrine (0.01 mg/kg per dose) was administered to a maximum of four doses. Hemodynamic parameters were measured throughout the experiment.

RESULTS: Animals were divided into survivors and nonsurvivors. End-diastolic and developed pressures declined after epinephrine administration in the survivor group. $\mathrm{d} p / \mathrm{d} t$ min was significantly higher in the survivor group whereas $\mathrm{dp} /$ $\mathrm{d} t$ max showed no significant differences. Epinephrine had no effect on either heart rate or cardiac output in both groups. Ejection fraction increased after epinephrine with no significant difference between groups.

CONCLUSION: Epinephrine did not affect survival rates or return of spontaneous circulation in our postnatal porcine model of neonatal asphyxia.

\footnotetext{
A
} sphyxia, a condition of impaired gas exchange with simultaneous hypoxia and hypercapnia leading to a mixed metabolic and respiratory acidosis, is the most common reason that newborns fail to make a successful fetal-to-neonatal transition $(1,2)$. Asphyxia could result from failure of placental gas exchange before delivery, secondary to an acute blood loss (e.g., abruption placentae) or deficient pulmonary gas exchange immediately after birth (e.g., respiratory distress syndrome) (1,2). Asphyxia depresses myocardial function leading to cardiogenic shock, pulmonary hypertension, peripheral and mesenteric vasoconstriction and hypoperfusion, acute renal failure, and increased blood flow to central organs such as brain and myocardium. In the clinical setting, newborn infants can present with mild, moderate, or severe asphyxia. Severe asphyxia presents with bradycardia or asystole at birth. Current resuscitation guidelines recommend chest compressions (CC) if the heart rate remains $<60 /$ min despite adequate ventilation with supplementary oxygen for $30 \mathrm{~s}^{3}$. Further, once CC are given, a vascular access should be established for epinephrine administration $^{3}$. Other than increasing the heart rate, heart contractility, and myocardial relaxation rate via stimulating $\beta$-receptors, epinephrine has also been shown to cause vasoconstriction of both peripheral and coronary arteries by activating $\alpha$-receptors $(3,4)$. However, both the effectiveness and safety for using epinephrine during resuscitation are still debatable (3-5). There is currently a lack of data evaluating the hemodynamic effects of epinephrine during neonatal cardiopulmonary resuscitation. We aimed to examine the hemodynamic changes after epinephrine administration during neonatal chest compression by using a porcine model of neonatal asphyxia.

\section{METHODS}

Twenty-four newborn mixed breed piglets (1-4 days of age, weighing $1.7-2.3 \mathrm{~kg}$ ) were obtained on the day of experimentation from the University Swine Research Technology Centre. All experiments were conducted in accordance with the guidelines and approval of the Animal Care and Use Committee (Health Sciences), University of Alberta and presented according to the ARRIVE guidelines (6).

\section{Animal Preparation}

Piglets were instrumented as previously described with modifications $(7,8)$. Following the induction of anesthesia using isoflurane, piglets were intubated via a tracheostomy, and pressure-controlled ventilation (Sechrist infant ventilator, model IV-100; Sechrist Industries, Anaheim, CA) was commenced at a respiratory rate of 16-20 breaths/min and pressure of $20 / 5 \mathrm{cmH}_{2} \mathrm{O}$. Oxygen saturation was kept within 90-100\%, glucose level and hydration were maintained with an intravenous infusion of $10 \%$ dextrose at $10 \mathrm{ml} / \mathrm{kg} / \mathrm{h}$. During the experiment, anesthesia was maintained with intravenous propofol $(5-10 \mathrm{mg} / \mathrm{kg} / \mathrm{h})$ and morphine $(0.1 \mathrm{mg} / \mathrm{kg} / \mathrm{h})$. Additional doses of propofol (1-2 mg/kg) and morphine (0.05-0.1 mg/kg) were

\footnotetext{
${ }^{1}$ Division of Neonatology, Department of Pediatrics, Pediatric Intensive Care and Neuropediatrics, Medical University Vienna, Vienna, Austria; ${ }^{2}$ Centre for the Studies of Asphyxia and Resuscitation, Neonatal Research Unit, Royal Alexandra Hospital, Edmonton, Alberta, Canada; ${ }^{3}$ Department of Pediatrics, University of Alberta, Edmonton, Alberta, Canada. Correspondence: Georg M. Schmölzer (georg.schmoelzer@me.com)

Received 24 July 2017; accepted 27 November 2017; advance online publication 10 January 2018. doi:10.1038/pr.2017.316
} 


\section{Articles | wagner et al.}

also given as needed. The piglet's body temperature was maintained at $38.5-39.5^{\circ} \mathrm{C}$ using an overhead warmer and a heating pad.

\section{Hemodynamic Parameters}

A 5-French Argyle (Klein-Baker Medical, San Antonio, TX) doublelumen catheter was inserted via the right external jugular vein for administration of fluids and medications. A 5-French Argyle singlelumen catheter was inserted below the right renal artery via the femoral artery for continuous arterial blood pressure monitoring in addition to arterial blood gas measurements. A Millar catheter (MPVS Ultra, ADInstruments, Houston, TX) was inserted into the left ventricle (LV) via the left common carotid artery for continuous measurement of left ventricular pressure, including composite and segmental volumes, which served as a surrogate for cardiac output. Because of the size difference between the Millar catheter and LV longitudinal axis, which poses a limitation for the accuracy of in vivo volume measurement, an alpha factor $=0.46$, based on comparison between Millar's recording and direct echocardiographic measurements in three piglets, was used to correct the conductance volume. The right common carotid artery was also exposed and encircled with a real-time ultrasonic flow probe $(2 \mathrm{~mm}$; Transonic Systems, Ithaca, NY) to measure blood flow.

Piglets were placed in supine position and allowed to recover from surgical instrumentation until baseline hemodynamic measures were stable. Ventilator rate was adjusted to keep the partial arterial $\mathrm{CO}_{2}$ between 35 and $45 \mathrm{~mm} \mathrm{Hg}$, as determined by periodic arterial blood gas analysis. Mean arterial pressure, heart rate, and percutaneous oxygen saturation were continuously measured and recorded throughout the experiment with a Hewlett Packard 78833B monitor (Hewlett Packard, Palo Alto, CA).

\section{Experimental Protocol}

Piglets were randomized into three experimental groups: 3:1 compression:ventilation ratio (C:V) $(3: 1, n=8)$; CC during sustained inflation (SI) at a rate of 90 CC per minute (SI+CC90, $n=8)$, or CC during SI at a rate of 120 CC per minute (SI+CC120, $n=8)(7)$. After surgical instrumentation and stabilization, piglets were exposed to $50 \mathrm{~min}$ of normocapnic hypoxia. Hypoxia was followed by asphyxia until the heart rate decreased to $25 \%$ of baseline, which was achieved by disconnecting the ventilator and clamping the endotracheal tube. Ten seconds after the heart rate reached $25 \%$ of baseline, positive pressure ventilation (PPV) was commenced for $30 \mathrm{~s}$ with a Neopuff T-Piece (Fisher \& Paykel, Auckland, New Zealand). The default settings were a peak-inflating pressure of $30 \mathrm{cmH}_{2} \mathrm{O}$, a positive end expiratory pressure of $5 \mathrm{cmH}_{2} \mathrm{O}$, and a gas flow of $8 \mathrm{l} / \mathrm{min}$. CC were performed using the two-thumb encircling technique by a single operator (ML) in all piglets. A metronome was used to achieve the targeted CC rate. After $30 \mathrm{~s}$ of CC, $100 \%$ oxygen was commenced. Epinephrine $(0.01 \mathrm{mg} / \mathrm{kg}$ per dose) was administered intravenously 1 min after the start of PPV, and administered every minute as needed if no increase in heart rate or return of spontaneous circulation (ROSC) was observed despite adequate ventilation and CC. Epinephrine was administered to a maximum of four doses. ROSC was defined as an unassisted heart rate $\geq 100 \mathrm{bpm}$ after $15 \mathrm{~s}$. After ROSC, piglets were allowed to recover for $4 \mathrm{~h}$ before the piglets were killed with an intravenous overdose of pentobarbital (100 mg/kg).

\section{Data Collection and Analysis}

Demographics of study piglets were recorded. Hemodynamic parameters including heart rate, mean/diastolic/systolic arterial pressure, stroke volume, cardiac output, ejection fraction, $\mathrm{d} p / \mathrm{d} t$ $\min / \mathrm{max}$, and Tau were continuously recorded using LabChart programming software (ADInstruments, Houston, TX). A 10-s epoch of pressure-volume $(P-V)$ loop parameters prior to hypoxia was used as the baseline. Ten-second epochs of $P-V$ loops (i) before PPV, (ii) after the start of CC, (iii) before each dose of epinephrine (max of four doses), and (iv) after each dose of epinephrine were analyzed. Epochs during $\mathrm{CC}$ and epinephrine were three consequent
CCs. Examples for different phases of PV loops are depicted in Figure 1.

Data are presented as mean \pm standard deviation (SD) for normally distributed continuous variables and median (interquartile range) when the distribution was skewed. Data were tested for normality and compared using one-way ANOVA, Student's $t$ test for parametric, and Mann-Whitney $U$-test for nonparametric comparisons of continuous variables, as appropriate. Post hoc analysis was performed using Tukey test. $P$ values are two sided and $P<0.05$ was considered statistically significant. Statistical analyses were performed with SigmaPlot (Systat Sofyware, San Jose, CA).

\section{RESULTS}

Based on the outcome of resuscitation, animals were divided into two groups: survivors who successfully achieved ROSC during resuscitation $(n=16)$ and nonsurvivors who did not achieve ROSC $(n=8)$. There were no significant differences in age, body weight, and cardiac function before hypoxia/asphyxia between these two groups (Table 1). During resuscitation, all nonsurvivor piglets received four doses of epinephrine, whereas only $50 \%$ of survivors received epinephrine ( $n=6$ with one dose, $n=1$ with three doses, and $n=1$ with four doses). As both the graph presentation and statistical analyses were obscured by the fourth dose of epinephrine in the survivor group, this data point was excluded.

The changes in arterial blood gases for two study groups at baseline and before resuscitation are summarized in Table 2. There was no significant difference in any parameters between survivors and nonsurvivors at these two different time points.

Figure 2 summarizes the changes in ventricular pressure during resuscitation. The ventricular pressures significantly declined from baseline to asphyxia with a similar recovery after CC for both groups (Figure 2a). After epinephrine administration, the developed pressures declined below the baseline value in survivors (Figure 2a). In contrast, in nonsurvivors, the developed pressure increased slightly after each dose of epinephrine and remained significantly higher throughout the resuscitation period when compared with survivors (Figure 2a).

As shown in Figure $\mathbf{2} \mathbf{b}$, the pattern of changes in endsystolic pressure was similar to the developed pressure pattern, and the values of the nonsurvivor group were significantly higher than that of the survivor group after receiving epinephrine. In contrast, the end-diastolic pressures of both groups increased after hypoxia/asphyxia with a further increase after CC. The end-diastolic pressure declined after epinephrine administration in the survivor group, whereas the pressures remained higher in the nonsurvivor group (Figure 2c). However, there was no statistically significant difference between these two groups.

Figure 3 summarizes changes from normoxic baseline in $\mathrm{dp} / \mathrm{dt} \max$ and $\mathrm{dp} / \mathrm{dt} \min$ on survivors and nonsurvivors during resuscitation. The $\mathrm{d} p / \mathrm{d} t \max$ and $\mathrm{d} p / \mathrm{d} t$ min represent the maximum and minimum rate of pressure change in the ventricle and have generally been used as an index of ventricular performance. The $\mathrm{d} p / \mathrm{d} t \max$ significantly decreased after hypoxia/asphyxia and increased after CC 


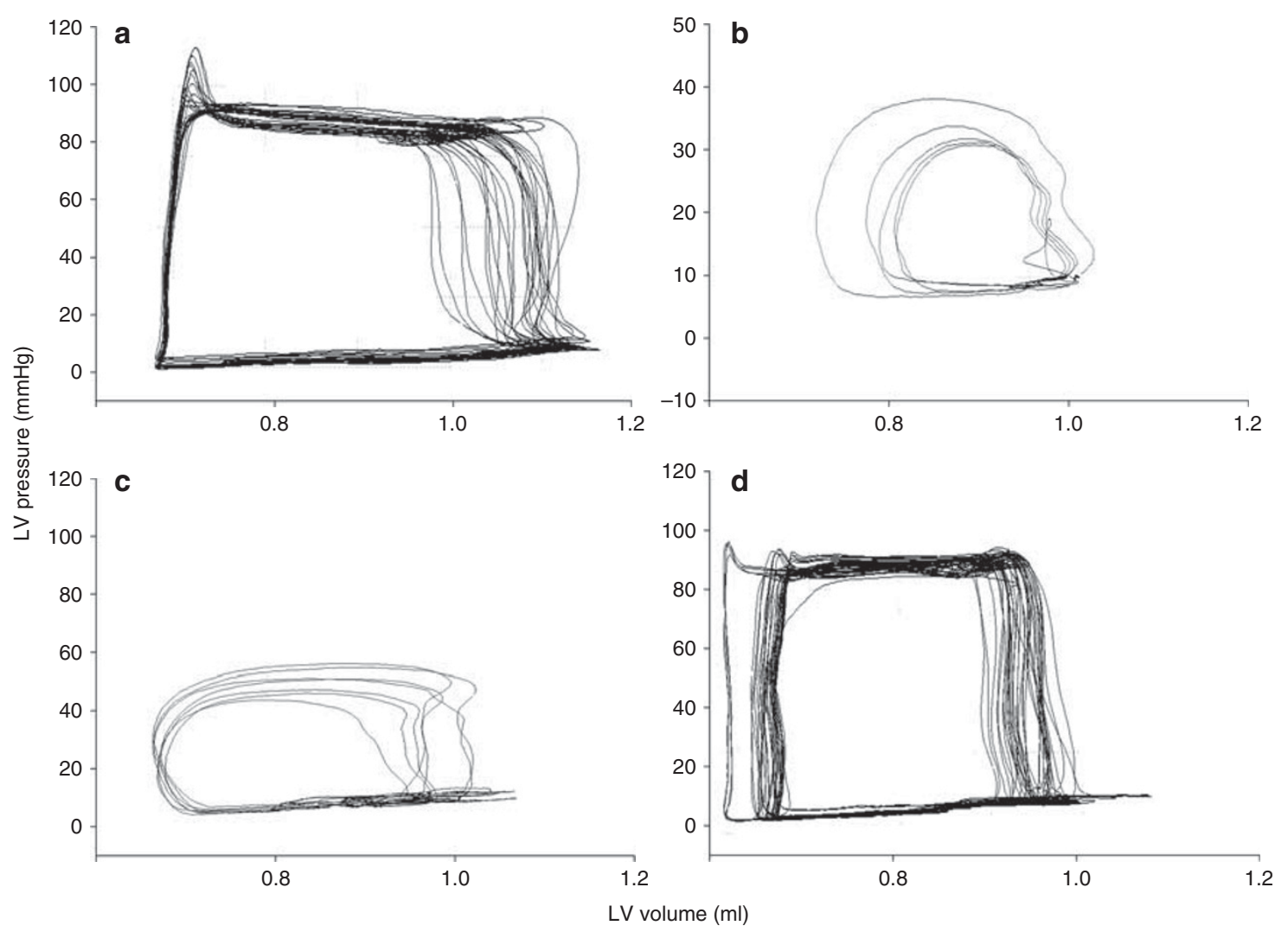

Figure 1. Examples of PV loops change during (a) baseline, (b) before PPV, (c) during CPR and (d) after ROSC. Please note that figure b is on different Y-scale for clarity.

Table 1. Baseline characteristics

\begin{tabular}{|c|c|c|c|}
\hline & Survivor $(n=16)$ & Nonsurvivor $(n=8)$ & $P$ value \\
\hline Age (days) & $2.0(1.5-3.5)$ & $2.5(2.0-3.8)$ & 0.72 \\
\hline Weight (kg) & $2.0(1.9-2.1)$ & $2.1(1.8-2.3)$ & 0.53 \\
\hline Cardiac output (ml/min) & $454(355-604)$ & 446 (407-604) & 0.81 \\
\hline Mean arterial pressure $(\mathrm{mm} \mathrm{Hg})$ & $74(65-74)$ & 75 (67-91) & 0.25 \\
\hline Developed pressure $(\mathrm{mm} \mathrm{Hg})$ & $47.1(42.5-49.9)$ & $38.9(30.4-52.0)$ & 0.23 \\
\hline End-systolic pressure $(\mathrm{mm} \mathrm{Hg})$ & $85.9(74.3-95.6)$ & $85.5(70.8-93.5)$ & 0.64 \\
\hline End-diastolic pressure $(\mathrm{mm} \mathrm{Hg})$ & $6.6(4.1-11.1)$ & $7.1(4.4-9.9)$ & 0.93 \\
\hline $\mathrm{d} p / \mathrm{d} t \max (\mathrm{mm} \mathrm{Hg} / \mathrm{s})$ & $4,605(4,246-5,955)$ & $4,733(3,719-5,756)$ & 0.75 \\
\hline
\end{tabular}

Data are presented as median (IQR).

(Figure 3a). It remained at a similar level after epinephrine administration and we observed no significant difference between both groups. The pattern of changes in $\mathrm{d} p / \mathrm{d} t \min$ was similar to changes in $\mathrm{d} p / \mathrm{d} t \max$ (Figure 3b). As compared with nonsurvivors, the $\mathrm{dp} / \mathrm{dt}$ min of survivors was higher after CC and remained significantly higher throughout the resuscitation period.
Figure 4 shows the changes in heart rate, cardiac output, ejection fraction, and tau between survivors and nonsurvivors during resuscitation. Changes in heart rate and cardiac output were similar between survivors and nonsurvivors. They decreased significantly before PPV and recovered after CC (Figures $4 \mathbf{a}$ and $\mathbf{b}$ ). Administration of epinephrine had no effect on either heart rate or cardiac output; there was no 
Articles | Wagner et al.

Table 2. Arterial blood gases at baseline and before resuscitation

\begin{tabular}{|c|c|c|c|c|}
\hline & \multicolumn{2}{|c|}{ Baseline } & \multicolumn{2}{|c|}{ Before resuscitation } \\
\hline & Survivor $(n=16)$ & Nonsurvivor $(n=8)$ & Survivor $(n=16)$ & Nonsurvivor $(n=8)$ \\
\hline $\mathrm{pH}$ & $7.35(7.31-7.40)$ & 7.37 (7.33-7.40) & $6.84(6.72-6.90)$ & $6.95(6.76-6.98)$ \\
\hline $\mathrm{PCO}_{2}(\mathrm{~mm} \mathrm{Hg})$ & $40.2(37.1-42.4)$ & $37.8(36.3-43.6)$ & $67.2(58.5-83.9)$ & $69.2(52.2-89.0)$ \\
\hline $\mathrm{HCO}_{3}(\mathrm{mmol} / \mathrm{l})$ & $22.4(20.3-24.3)$ & $25.2(20.3-29.1)$ & $11.4(9.9-12.3)$ & $13.3(10.5-14.7)$ \\
\hline Base excess (mmol/l) & $-3(-6$ to -1.5$)$ & $-3(-5 \sim 4)$ & $-23(-25$ to -21$)$ & $-21(-24$ to -17$)$ \\
\hline Lactate (mmol/l) & $3.5(2.5-4.3)$ & $4.2(2.2-6.1)$ & $14.0(12.8-14.6)$ & $14.2(13.9-15.8)$ \\
\hline
\end{tabular}

Data are presented as median (IQR)
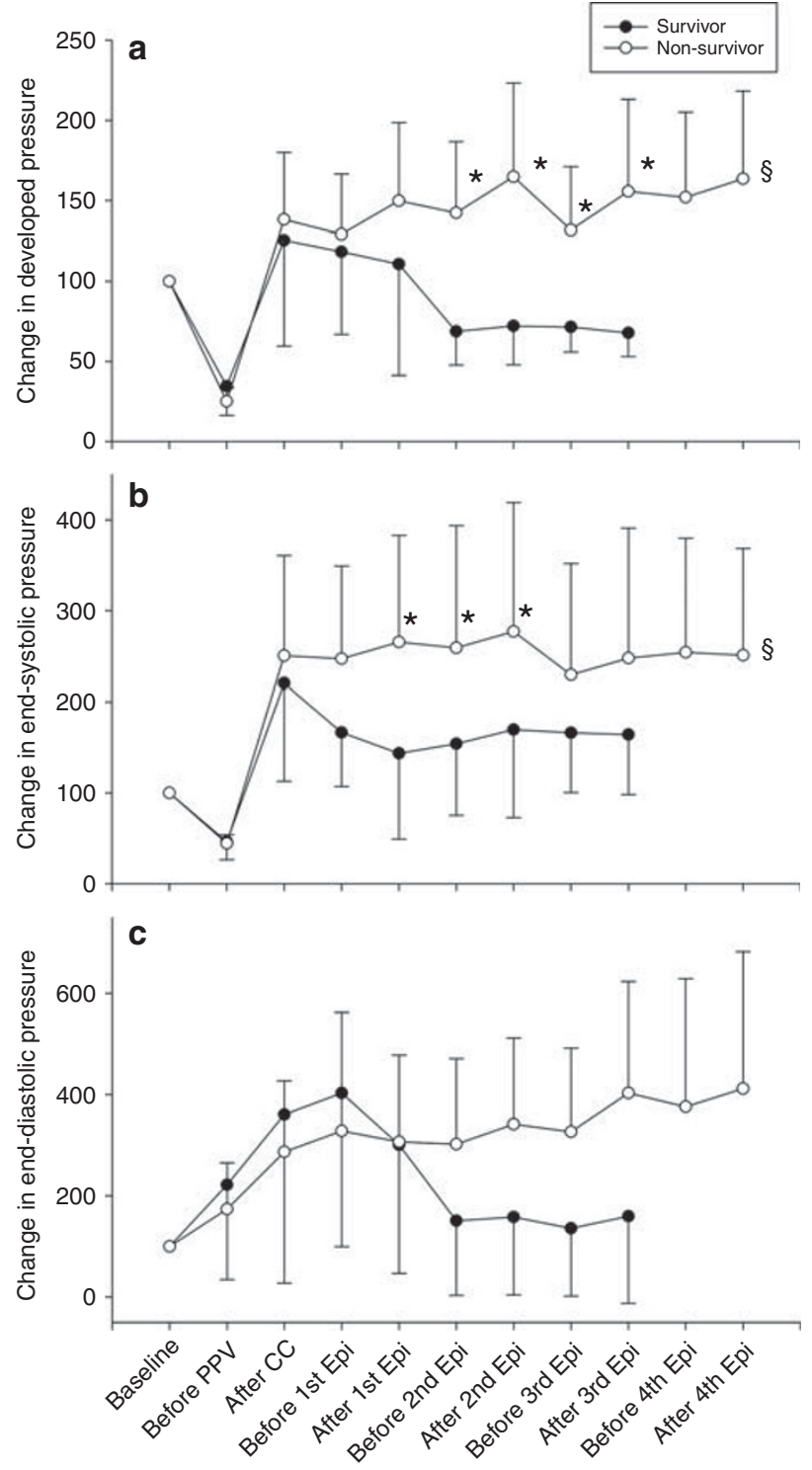

Figure 2. Percentage changes from normoxic baseline in (a) developed blood pressure, (b) end-systolic and (c) end-diastolic pressure on nonsurvivor (white dots) and survivor (dark dots) during and after resuscitation. Each point epresents mean \pm SD. §Significantly different from survivor group at the same time point, $p<0.05$ (Tukey).

significant difference between survivors and nonsurvivors throughout the resuscitation period (Figures $4 a$ and b). Figure $4 \mathrm{c}$ shows that ejection fraction significantly declines
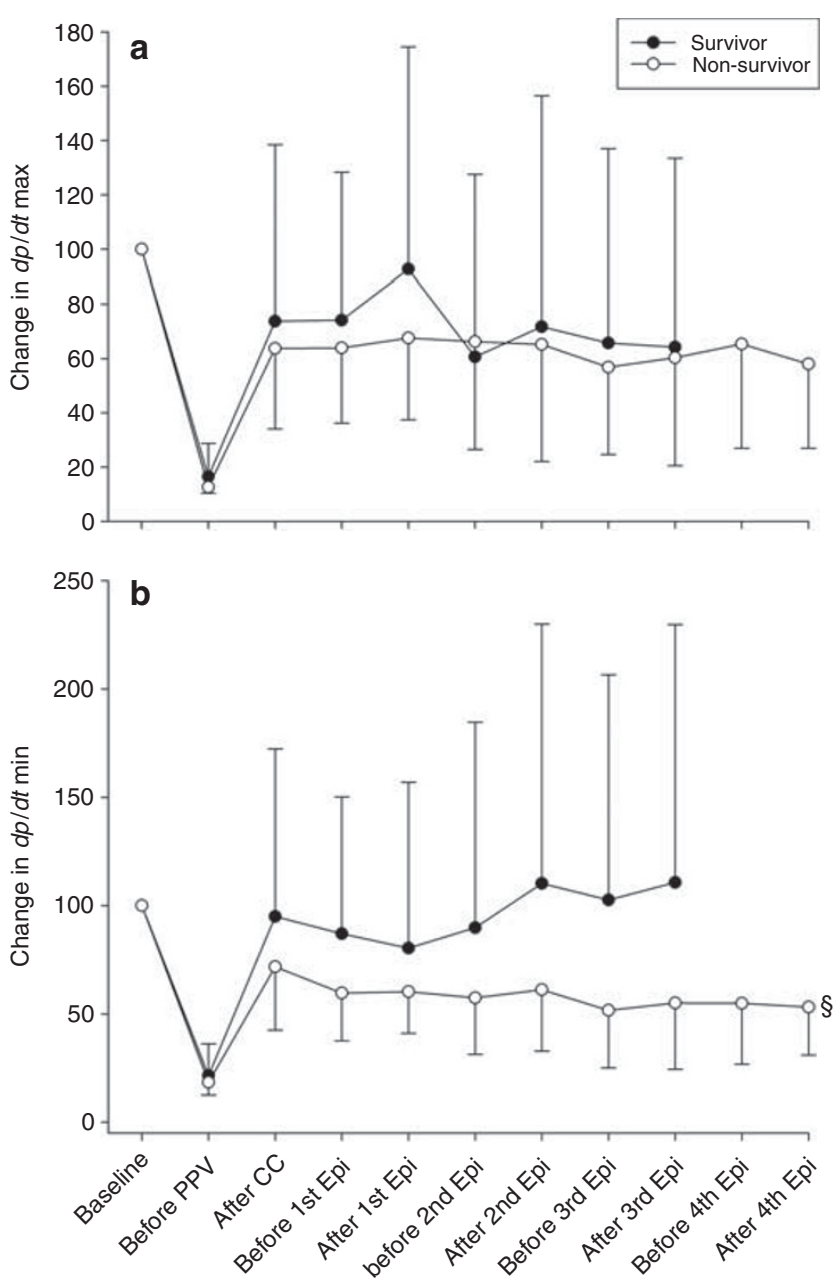

Figure 3. Percentage changes from normoxic baseline in (a) $\mathrm{d} p / \mathrm{d} t$ max and (b) $\mathrm{d} p / \mathrm{d} t$ min on non-survivor (white dots) and survivor (dark dots) during and after resuscitation. Each point represents mean \pm SD. $\S$ Significantly different from survivor group throughout experimental period, $p<0.05$ (2-way ANOVA).

after hypoxia/asphyxia and increased with CC. Administration of epinephrine had no effects on ejection fraction and no significant difference between survivors and nonsurvivors was observed.

Isovolumic relaxation constant (Tau), represents the exponential decay of the ventricular pressure during 

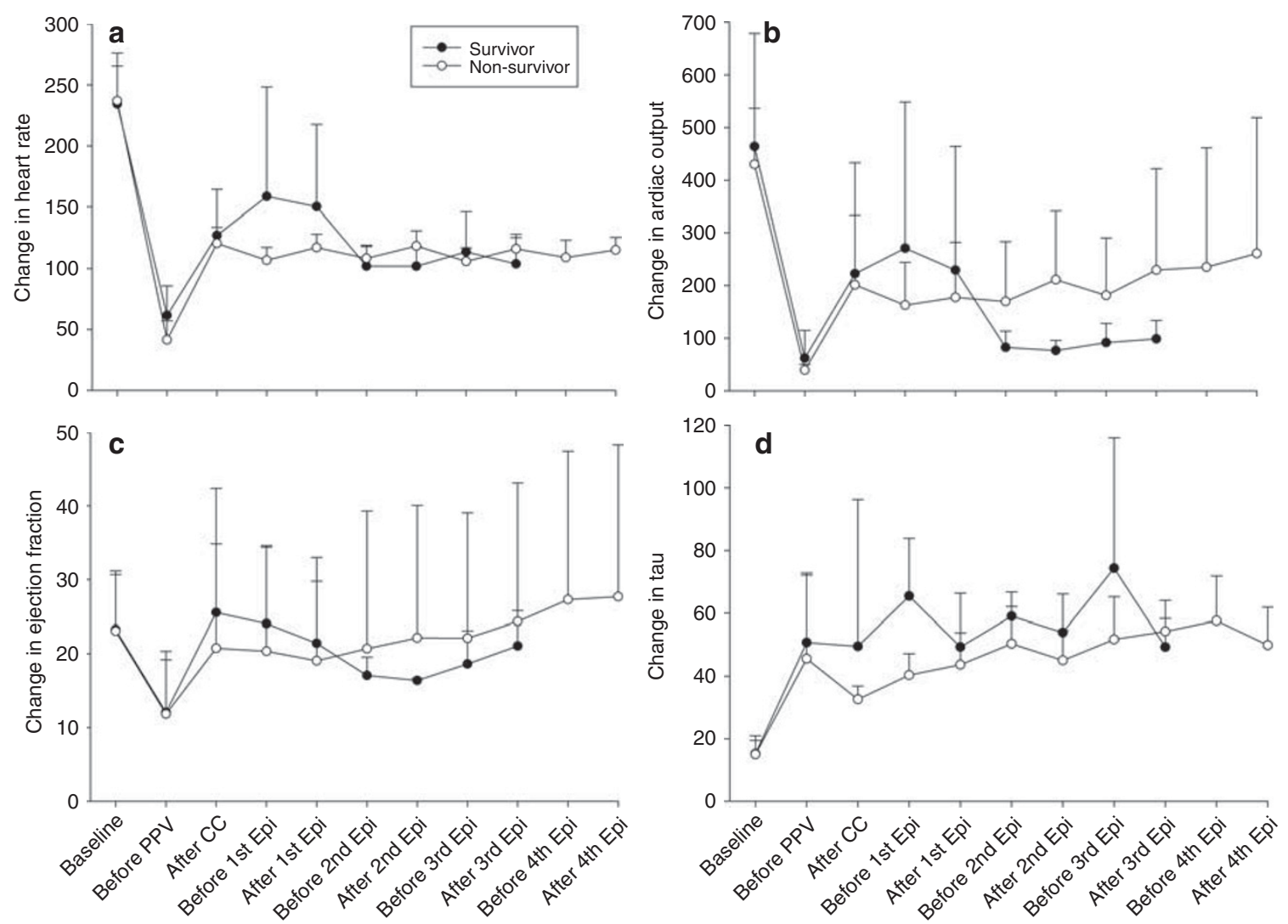

Figure 4. Changes in (a) heart rate, (b) cardiac output, (c) ejection fraction and (d) tau on non-survivor (white dots) and survivor (dark dots) during and after resuscitation. Each point represents mean \pm SD.

isovolumic relaxation and is calculated with Gantz method. Tau of survivors was higher when compared with baseline before PPV and Tau remained about the same level throughout the resuscitation period (Figure 4d). However, the pattern of Tau in nonsurvivors was also similar and there was no significant difference between these two groups.

\section{DISCUSSION}

This is the first study to examine cardiac changes after epinephrine administration during neonatal CC in a porcine model of neonatal resuscitation using a Millar catheter. The findings of our study can be summarized as the following: (i) $50 \%$ of piglets did not require epinephrine administration for ROSC, (ii) epinephrine administration did not elicit significant chronotropic and inotropic effects, and (iii) might lower coronary perfusion pressure by decreasing diastolic function.

Epinephrine has been recommended during resuscitation to aid cardiovascular recovery (9). Its effects are dependent on the balance of each adrenergic receptor subtype, as well as its binding affinities at various conditions (10), and currently, its receptor distribution is poorly studied in newborn infants. Our study showed that there is a significant positive effect of PPV and CC on cardiac parameters responsible for ROSC, whereas epinephrine only exhibited a minor additional effect on the outcome of hypoxic piglets. In the current study, only $50 \%$ of survivors received epinephrine for achieving ROSC. As expected, we observed a decrease of heart rate and cardiac output in survivors and nonsurvivors after hypoxia, which was followed by an increase once CC were started. Even though there was no statistical difference, both heart rate and cardiac output were slightly higher within the survivor group (Figure 4). Administration of epinephrine caused a slight increase in ejection fraction (Figure 4), however, it did not further increase heart rate or cardiac output. We believe that acidosis might have aided in the ineffectiveness of epinephrine in improving ROSC (11). Our finding is similar to a study by Linner et al. (12), who also reported that epinephrine did not improve ROSC in their neonatal porcine model. In contrast, Sobotka et al. (13)reported that CC alone was not enough to restore ROSC in asphyxiated newborn lambs and that epinephrine was critical for increasing heart rate, carotid arterial pressure, and cerebral blood flow. Halling et al. suggested to start with higher first doses $(0.03 \mathrm{mg} / \mathrm{kg})$ of epinephrine as they described it superior at achieving ROSC with cumulative doses (14). This suggestion is supported by Vali et al. (15), who showed a high ROSC success rate after one high dose $(0.03 \mathrm{mg} / \mathrm{kg})$ of intravenous epinephrine .

Vasopressin might be an alternative catecholamine for asphyxiated newborns due to its greater vasoconstrictor effect 


\section{Articles | wagner et al.}

during acidosis (16). In adults, vasopressin has been suggested to be more effective than epinephrine in asystole cardiac arrest, which is commonly the result of severe asphyxia (17). In a neonatal porcine model of asphyxia, McNamara et al. (16) reported significantly higher survival rates after vasopressin administration compared with epinephrine.

A coronary perfusion pressure threshold of $20 \mathrm{~mm} \mathrm{Hg}$ is needed to achieve ROSC and might be achieved with the combination of CC, adequate ventilation, and epinephrine administration (18). Morgan et al. (18) compared a hemodynamic-directed chest compression technique with CC depth titrated to achieve a systolic blood pressure of $90 \mathrm{~mm} \mathrm{Hg}$, and vasopressors were only given if the coronary perfusion pressure was $<20 \mathrm{~mm} \mathrm{Hg}$ in asphyxiated piglets. Hemodynamic-directed CPR was associated with significantly increased coronary blood pressure, right atrial diastolic pressure, aortic diastolic pressure, and faster ROSC compared with the standard CPR. As compared with the nonsurvivor group, the $\mathrm{d} p / \mathrm{d} t$ min of the survivor group remained higher throughout the resuscitation period. Administration of epinephrine did not increase $\mathrm{d} p / \mathrm{d} t$ min in the nonsurvivor group. An increase in $\mathrm{d} p / \mathrm{d} t$ min is associated with an increase in diastolic function during isovolumic relaxation. Therefore, the faster ROSC in the survivor group was most likely due to better diastolic function. This is further supported by the observation that epinephrine maintained higher end-diastolic pressure in our nonsurvivor group. As coronary perfusion pressure is defined as diastolic pressure minus end-diastolic pressure, higher end-diastolic pressure will result in lower coronary perfusion pressure. In our study, end-diastolic pressure declined in the survivor group. This is similar to findings by Vali et al. (15), who reported that in their model of neonatal chest compression, epinephrine did not significantly elevate blood pressure or coronary perfusion pressure, and ROSC was achieved with low mean diastolic blood pressures $(\sim 10 \mathrm{~mm} \mathrm{Hg})$. The authors suggested that systolic blood pressures may be more predictive of ROSC as the lambs in their study achieved ROSC with low mean diastolic blood pressures $(\approx 10 \mathrm{~mm} \mathrm{Hg})$ (15).

Other than its ineffectiveness in improving ROSC in our present study, the use of epinephrine has also been linked to various undesired side effects. Epinephrine administration could lead to cerebral vascular injury and intraventricular hemorrhage in asphyxiated newborns due to rapid blood pressure changes because of prolonged hypertension, decreased cerebral blood flow, and tachycardia (13). A randomized clinical trial in children reported no improvement in ROSC but increased mortality after epinephrine administration (19). Similarly, Savani et al. (20) reported lower survival rates and higher incidence of intraventricular hemorrhage during delivery room resuscitation. This might be due to an increased susceptibility of catecholamineinduced toxicity of the neonatal myocardium compared with adults (21). Further studies are thus required to examine the benefits and risks for using epinephrine during resuscitation.

\section{Limitation}

Our use of a piglet asphyxia model is a considerable strength of this translational study because this model closely mimics delivery room events with a gradual onset of severe asphyxia leading to bradycardia. However, several limitations should be considered: The current model is one where the piglets have already undergone fetal-to-neonatal transition and piglets are sedated/anesthetized. In addition, piglets in our model were all intubated using a tightly secured endotracheal tube to prevent any endotracheal tube leak; this is different from a clinical situation where mask ventilation may be frequently used. Of note, giving $100 \%$ oxygen after $30 \mathrm{~s}$ of CC and the administration of epinephrine at $60 \mathrm{~s}$ after CC started and continued every minute, are not in line with the current resuscitation guidelines $(9,22)$. Although all piglets were resuscitated with the same protocol, they were randomized to three experimental study groups, which might have influenced the results.

\section{Conclusion}

Epinephrine did not increase survival rates or ROSC in a postnatal porcine model of neonatal asphyxia. Further studies are needed to examine the optimal vasopressor including optimal dose and route of administration during cardiopulmonary resuscitation.

\section{AUTHOR CONTRIBUTIONS}

Conception and design: G.M.S. and P.Y.C. Collection and assembly of data: G.M.S., E.S.L., T.F.L., M.L., M.O.R., P.Y.C., and M.W. Analysis and interpretation of the data: G.M.S., E.S.L., T.F.L., M.L., M.O.R., P.Y.C., M.W., and M.O. Drafting of the article: G.M.S., E.S.L., T.F.L., M.L., M.O.R., P.Y.C., M.W., and M.O. Critical revision of the article for important intellectual content: G.M.S., E.S.L., T.F.L., M.L., M.O.R., P.Y.C., M.W., and M.O. Final approval of the article: G.M.S., E.S. L., T.F.L., M.L., M.O.R., P.Y.C., M.W., and M.O.

\section{STATEMENT OF FINANCIAL SUPPORT:}

We thank the public for donating money to our funding agencies: E.S.L. was supported by Alberta Innovates-Health Solutions SummerStudentship. G.M.S. is a recipient of the Heart and Stroke Foundation/ University of Alberta Professorship of Neonatal Resuscitation, a National New Investigator of the Heart and Stroke Foundation Canada, and an Alberta New Investigator of the Heart and Stroke Foundation Alberta. We acknowledge the Neonatal Resuscitation Program Canada Grant Program within the Canadian Society of Pediatrics for supporting the study.

Disclosure: The authors declare no conflict of interest.

\section{REFERENCES}

1. Kapadia V, Wyckoff MH. Chest compressions for bradycardia or asystole in neonates. Clin Perinatol 2012;39:833-42.

2. Solevåg $\mathrm{AL}$, Cheung $\mathrm{P}-\mathrm{Y}$, O'Reilly $\mathrm{M}$, Schmölzer GM. A review of approaches to optimise chest compressions in the resuscitation of asphyxiated newborns. Arch Dis Child Fetal Neonatal Ed 2016;101: F272-6.

3. Pinto M, Solevåg AL, O'Reilly M, Aziz K, Cheung P-Y, Schmölzer GM. Evidence on adrenaline use in resuscitation and its relevance to newborn infants: a non-systematic review. Neonatology 2016;111:37-44.

4. Kapadia VS, Wyckoff MH. Epinephrine use during newborn resuscitation. Front Pediatr 2017;5:97.

5. Barber CA, Wyckoff MH. Use and efficacy of endotracheal versus intravenous epinephrine during neonatal cardiopulmonary resuscitation in the delivery room. Pediatrics 2006;118:1028-34. 
6. Kilkenny C, Browne WJ, Cuthill IC, Emerson M, Altman DG. Improving bioscience research reporting: the ARRIVE guidelines for reporting animal research. Osteoarthr Cartil 2012;20:256-60.

7. Li ES, Cheung P-Y, Lee T-F, Lu M, O'Reilly M, Schmölzer GM. Return of spontaneous circulation is not affected by different chest compression rates superimposed with sustained inflations during cardiopulmonary resuscitation in newborn piglets. PLoS ONE 2016;11:e0157249-14.

8. Li ES, Görens I, Cheung P-Y, et al. Chest compressions during sustained inflations improve recovery when compared to a 3:1 compression: ventilation ratio during cardiopulmonary resuscitation in a neonatal porcine model of asphyxia. Neonatology 2017;112:337-46.

9. Wyckoff MH, Aziz K, Escobedo MB, et al. Part 13: neonatal resuscitation: 2015 American Heart Association Guidelines update for cardiopulmonary resuscitation and emergency cardiovascular care (reprint). Pediatrics 2015;136 (Suppl 2): S196-218.

10. Weiner GM, Niermeyer S. Medications in neonatal resuscitation: epinephrine and the search for better alternative strategies. Clin Perinatol 2012;39:843-55.

11. Idris AH, Becker LB, Fuerst RS, et al. Effect of ventilation on resuscitation in an animal model of cardiac arrest. Circulation 1994;90:3063-9.

12. Linner R, Werner O, Perez-de-Sa V, Cunha-Goncalves D. Early adrenaline administration does not improve circulatory recovery during resuscitation from severe asphyxia in newborn piglets. Resuscitation 2012;83:1298-303.

13. Sobotka KS, Polglase GR, Schmölzer GM, Davis PG, Klingenberg C, Hooper SB. Effects of chest compressions on cardiovascular and cerebral hemodynamics in asphyxiated near-term lambs. Pediatr Res 2015;78: $395-400$.
14. Halling C, Sparks JE, Christie L, Wyckoff MH. Efficacy of intravenous and endotracheal epinephrine during neonatal cardiopulmonary resuscitation in the delivery room. J Pediatr 2017;185:232-6.

15. Vali $\mathrm{P}$, Chandrasekharan $\mathrm{P}$, Rawat $\mathrm{M}$, et al. Evaluation of timing and route of epinephrine in a neonatal model of asphyxial arrest. J Am Heart Assoc 2017;6:e004402-13.

16. McNamara PJ, Engelberts D, Finelli M, Adeli K, Kavanagh BP. Vasopressin improves survival compared with epinephrine in a neonatal piglet model of asphyxial cardiac arrest. Pediatr Res 2014;75:738-48.

17. Wenzel V, Krismer AC, Arntz HR, Sitter H, Stadlbauer KH, Lindner KH. A comparison of vasopressin and epinephrine for out-of-hospital cardiopulmonary resuscitation. N Engl J Med 2004;350:105-13.

18. Morgan RW, Kilbaugh TJ, Shoap W, et al. A hemodynamic-directed approach to pediatric cardiopulmonary resuscitation (HD-CPR) improves survival. Resuscitation 2017;111:41-7.

19. Perondi MBM, Reis AG, Paiva EF, Nadkarni VM, Berg RA. A comparison of high-dose and standard-dose epinephrine in children with cardiac arrest. N Engl J Med 2004;350:1722-30.

20. Savani M, Upadhyay K, Talati AJ. Characteristics and outcomes of very low birth weight infants receiving epinephrine during delivery room resuscitation. Resuscitation 2017;115:1-4.

21. Caspi J, Coles JG, Benson LN, et al. Age-related response to epinephrineinduced myocardial stress. A functional and ultrastructural study. Circulation 1991;84:III394-9.

22. Perlman JM, Wyllie J, Kattwinkel J, et al. Part 7: neonatal resuscitation: 2015 International Consensus on Cardiopulmonary Resuscitation and Emergency Cardiovascular Care Science With Treatment Recommendations (Reprint). Pediatrics 2015;136 (Suppl 2): S120-66. 